\title{
Coexistence of Atrioventricular Nodal Reentrant Tachycardia with Other Forms of Arrhythmias
}

\author{
Christiana Schernthaner Franz Danmayr Bernhard Strohmer \\ Department of Cardiology, Paracelsus Private Medical University, Salzburger Landeskliniken, Salzburg, Austria
}

\section{Key Words}

Supraventricular tachycardia · Atrioventricular nodal reentrant tachycardia - Electrophysiological study .

Radiofrequency catheter ablation · Atrial fibrillation

\begin{abstract}
Objective: The aim of this retrospective study was to investigate the association of atrioventricular nodal reentrant tachycardia (AVNRT) with other forms of arrhythmia in individual patients and its consequences for treatment. Subjects and Methods: This study comprised 493 consecutive patients aged $16-88$ years ( 296 women and 197 men) who were diagnosed with a form of AVNRT via a standard 4-catheter electrophysiological study (EPS). Patients were clinically followed (range $0.5-12$ years) at a single center. Results: Coexistence of AVNRT with other types of tachycardias was observed in 197 (40\%) patients. Atrial fibrillation was found most frequently in 94 (19\%) patients as follows: focal atrial tachycardia, $n=40(8 \%)$; atrial flutter, $n=32(6 \%)$, and AV reentrant tachycardia, $n=22(4 \%)$. Double tachycardia was present in 140 (30\%) patients, and more than 2 different types of tachycardias were present in 57 (12\%) patients. Transitions between AVNRT and other tachycardias occurred in 25 (5\%) patients. Two or more tachycardias were
\end{abstract}

ablated in 42 (9\%) patients. The majority of patients were free of symptoms at the first follow-up, whereas 130 (26\%) patients reported a variety of symptoms. Conclusion: Coexistence of AVNRT with other types of arrhythmias was a common finding among these patients. The most frequently observed double tachycardia was the combination of AVNRT with atrial tachyarrhythmias, such as atrial fibrillation, with a potential significance for further patient management.

(c) 2014 S. Karger AG, Basel

\section{Introduction}

Atrioventricular nodal reentrant tachycardia (AVNRT) is a common arrhythmia of supraventricular tachycardias (SVT) in clinical electrophysiological practice. Coexistence of more than one SVT in an individual patient is a well-known but infrequent phenomenon [17]. The aim of this study was to investigate the association of AVNRT with other forms of arrhythmias in individual patients at the time of electrophysiological study (EPS), the consequences for treatment, and the clinical relevance of additionally induced arrhythmias, particularly as far as the clinical outcome was concerned.

\begin{tabular}{ll}
\hline KARGER & $\begin{array}{l}\text { ( ) 2014 S. Karger AG, Basel } \\
1011-7571 / 14 / 0236-0543 \$ 39.50 / 0 \quad \text { Karger }\end{array}$ \\
$\begin{array}{l}\text { E-Mail karger@karger.com } \\
\text { www.karger.com/mpp }\end{array}$ & $\begin{array}{l}\text { Thisis an Open Access article licensed under the terms of the } \\
\text { Creative Commons Attribution-NonCommercial 3.0 Un- } \\
\text { ported license (CC BY-NC) (www.karger.com/OA-license), } \\
\text { applicable to the online version of the article only. Distribu- } \\
\text { tion permitted for non-commercial purposes only. }\end{array}$
\end{tabular}

Christiana Schernthane

Department of Cardiology, Paracelsus Private Medical University

Salzburger Landeskliniken, Müllner Hauptstrasse 48

AT-5020 Salzburg (Austria)

E-Mail c.schernthaner@salk.at 
Table 1. Clinical and electrophysiological characteristics of all patients with AVNRT $(\mathrm{n}=493)$

\begin{tabular}{lc} 
Gender & \\
Male & $198(40)$ \\
Female & $296(60)$ \\
Age, years & $53 \pm 13(16-88)$ \\
Arterial hypertension & $104(21)$ \\
Coronary artery disease & $42(9)$ \\
Cardiomyopathy & $12(2)$ \\
Clinical presentation & \\
Palpitations & $487(99)$ \\
Syncope & $5(1)$ \\
Sudden cardiac death survivor & $1(0)$ \\
ECG documentation prior to EPS & $405(82)$ \\
Narrow QRS tachycardia & $5(1)$ \\
Wide \pm narrow QRS tachycardia & \\
Form of AVNRT & $454(92)$ \\
Typical & $26(5)$ \\
Atypical & $13(3)$ \\
Typical and atypical & \\
Induction of ANVRT & $320(65)$ \\
At baseline & $67(14)$ \\
With atropine & $106(22)$ \\
With orciprenaline \pm atropine & \\
Inducibility of AVNRT & $469(95)$ \\
Sustained form & $24(5)$ \\
Nonsustained form & $295(60)$ \\
Endpoint of ablation & $198(40)$ \\
Slow-pathway modification & \\
Slow-pathway elimination & \\
\hline
\end{tabular}

Values represent $\mathrm{n}(\%)$ or mean $\pm \mathrm{SD}$ (range).

\section{Subjects and Methods}

This retrospective study comprised a total of 493 consecutive patients who were referred to our institution for ablation of SVT from March 2001 to November 2012. The referral for invasive EPS was based either on a documented electrocardiogram or on recurrent palpitations suggestive of any type of SVT. A cardiac electronic device had been previously implanted in 9 patients (insertable loop recorder, $\mathrm{n}=2$; pacemaker, $\mathrm{n}=1$, and implantable cardioverter defibrillator, $\mathrm{n}=6$ ). After having obtained written informed consent, an EPS was performed on patients in a fasted state after discontinuation of all antiarrhythmic drugs for at least 3 days. Conscious sedation was achieved with midazolam and fentanyl. A 4-catheter EPS was carried out. Three quadripolar electrode catheters were introduced from the right and left femoral veins and placed in the high right atrium, His bundle area, and right ventricle. A decapolar catheter was inserted from the right internal jugular vein and placed in the coronary sinus. Standard electrophysiological pacing techniques and criteria were used to diagnose the type of SVT $[8,9]$ and to distinguish between typical and atypical forms of AVNRT [10]. If tachycardia could not be induced in the baseline state, orciprenaline and/or atropine was administered to facilitate the induction of tachycardia. The usual dosage of atropine was a bolus injection of $0.5 \mathrm{mg}$ repeated up to
Table 2. Frequency of different arrhythmias coexisting with AVNRT at the time of electrophysiological testing $(\mathrm{n}=197 ; 40 \%)$

\begin{tabular}{lc}
\hline Arrhythmia forms coexisting with AVNRT & $\mathrm{n}$ \\
\hline Atrial fibrillation & 94 \\
Atrial tachycardia & 40 \\
Atrial flutter & 32 \\
Atrioventricular reciprocating tachycardia & 22 \\
Right ventricular outflow tract tachycardia/extrasystole & 21 \\
Intra-atrial reentry tachycardia & 19 \\
Sinus node reentry tachycardia & 18 \\
Ventricular tachycardia & 13 \\
Bradyarrhythmia & 4 \\
Junctional tachycardia & 3 \\
\hline
\end{tabular}

$2 \mathrm{mg}$, and for orciprenaline infusion a rate of $0.5 \mu \mathrm{g} / \mathrm{min}$ was used until the heart rate increased by $30 \%$.

A coexisting arrhythmia was diagnosed apart from AVNRT if it was sustained for at least $6 \mathrm{~s}$. If AVNRT was noninducible or unsustainable, empiric slow-pathway catheter ablation was performed only if a previous 12-lead surface ECG of the clinical arrhythmia was consistent with AVNRT and/or at least 2 atrioventricular nodal reentrant echos were reproducibly present during the EPS. Ablation of AVNRT was carried out using a bidirectional deflectable catheter with a 4-mm distal tip. Radiofrequency energy was delivered using a Stockert RF generator (Biosense Webster, Diamond Bar, Calif., USA) at $50 \mathrm{~W}$ limited to $60^{\circ} \mathrm{C}$ at the critical sites for termination of the arrhythmias [11-13]. A 3-dimensional mapping system (CARTO ${ }^{\mathrm{TM}}$, Biosense Webster, or NavX/ EnSite $^{\mathrm{TM}}$, St. Jude Medical) was implemented whenever reasonable to target the coexisting arrhythmia.

All patients were scheduled for a routine follow-up visit at our outpatient clinic after the ablation procedure. Later on, all patients were requested to get in contact with our institution whenever problems occurred that might be related to any type of arrhythmia. In the event of recurrent symptoms, the patients were provided with either continuous electrocardiographic Holter monitoring for up to $48 \mathrm{~h}$ or an external loop recorder to document the rhythm at the time of the symptoms. In case of an implanted cardiac electronic device, such as an insertable loop recorder, a pacemaker, or an implantable cardioverter defibrillator, stored intracardiac electrograms were used to determine the type of arrhythmia.

\section{Statistical Analysis}

Data for continuous variables were expressed as means \pm SD or medians if appropriate. Univariate analysis was performed using Fisher's exact test to determine differences for categorical data. $\mathrm{p}<0.05$ was considered statistically significant.

\section{Results}

All of the 493 consecutive patients were diagnosed with a form of AVNRT. The mean age was $53 \pm 13$ years (range 16-88), with a higher prevalence of women $(\mathrm{n}=$ 


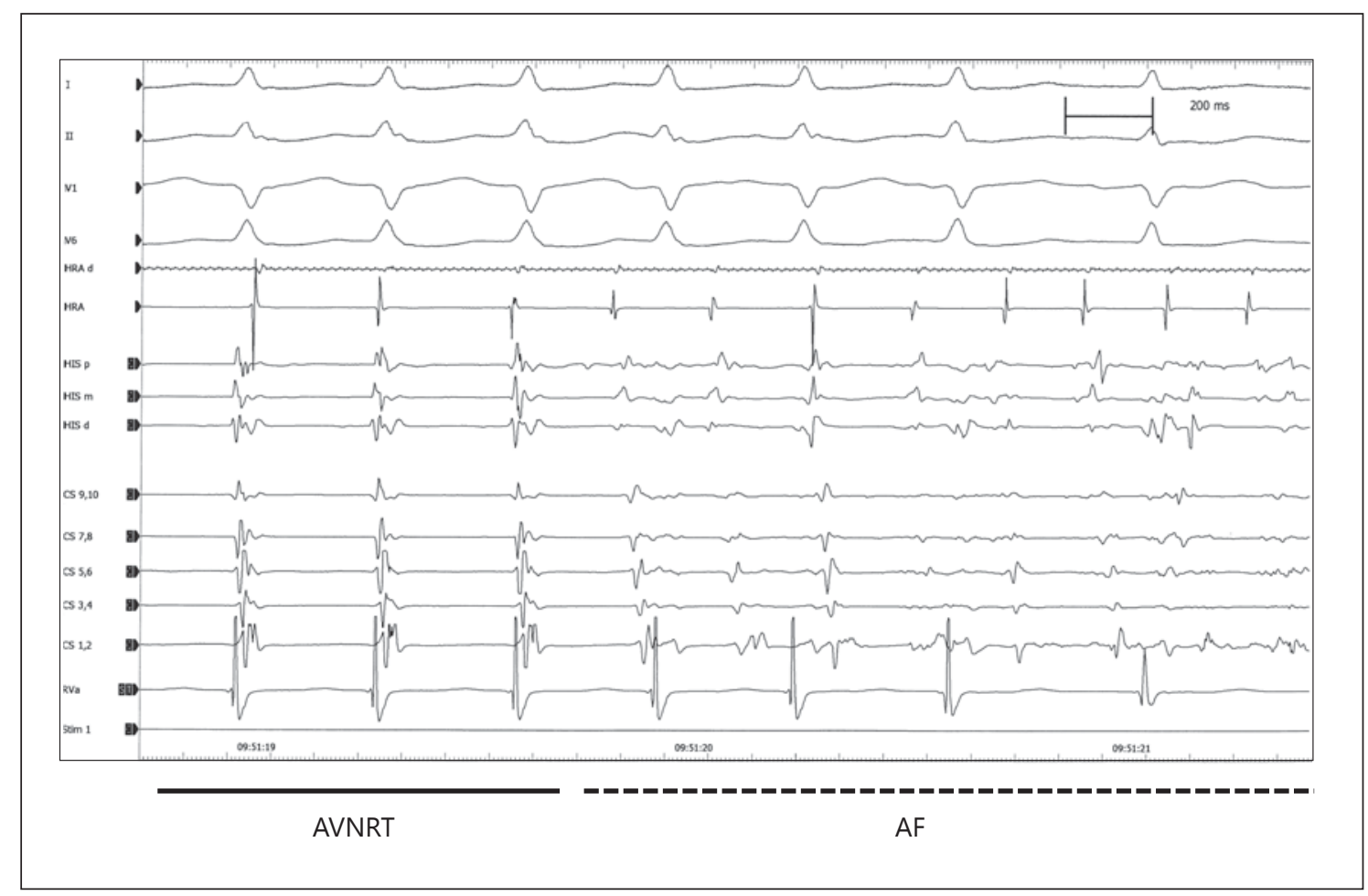

Fig. 1. Transition from ongoing typical AVNRT (CL 320 ms; P-on$\mathrm{R}$ pattern) to $\mathrm{AF}$ with a disorganized irregular intracardiac activation pattern. Displayed are surface leads I, II, V1, and V6, and bipolar electrogram recordings from the high right atrium (HRA distal, proximal), the His bundle (HIS proximal to distal), the coronary sinus (CS 9, 10 proximal to CS 1,2 distal), and the right ventricular apex (RVa).
$296 ; 60 \%)$ than men ( $\mathrm{n}=197 ; 40 \%)$. The majority of the patients had no significant underlying structural heart disease. Arterial hypertension was present in 104 (21\%), coronary artery disease was found in 42 (9\%), and cardiomyopathy was present in $12(2 \%)$ patients. The clinical and electrophysiological characteristics are outlined in table 1 . Most of the patients $(\mathrm{n}=405 ; 82 \%)$ were referred for EPS following documentation of a regular narrow QRS tachycardia, whereas only a few patients $(\mathrm{n}=5,1 \%)$ presented either a wide and/or a narrow complex tachycardia ahead of the invasive procedure. Syncope related to rapid tachycardia was reported in $5(1 \%)$ patients. One patient had a history of successful resuscitation following sustained tachycardia during physical exertion with a subsequent hemodynamic collapse.

Typical (slow-fast) AVNRT was diagnosed in 454 (92\%) patients, atypical AVNRT was diagnosed in 26 (5\%) patients, and both forms of AVNRT were diagnosed in $13(3 \%)$ patients (table 1). AVNRT was inducible in a sustained form in 469 (95\%) patients and in a nonsustained form in $24(5 \%)$ patients. In $65 \%(n=320)$ of the patients AVNRT was already inducible at baseline, whereas in $35 \%$ a pharmacological provocation such as with atropine $(n=67)$, orciprenaline $(n=10)$, or a combination of atropine and orciprenaline $(n=96)$ was required for induction. Overall, the coexistence of AVNRT with other tachycardias was observed in 197 (40\%) patients. A 12-lead ECG documenting other types of arrhythmias besides AVNRT was available in only 5 of these patients prior to electrophysiological testing. Atrial fibrillation (AF) was the most common additional arrhythmia, inducible in 94 (19\%) patients, followed by focal atrial tachycardia in 40 (8\%), atrial flutter in $32(6 \%)$, AV reciprocating tachycardia in $22(4 \%)$, sinus node reentry tachycardia in $18(4 \%)$, intra-atrial reentry tachycardia in $19(4 \%)$, and junctional tachycardia in $3(1 \%)$ patients (table 2). The simultaneous occurrence of AVNRT with ventricular outflow tract extrasystole and/ or tachycardia was observed in 21 (4\%) patients. Concomitance of arrhythmias in the form of double tachycardia ( $\mathrm{n}=140 ; 28 \%)$ was more frequent than the combination of AVNRT with more than 2 different types of 


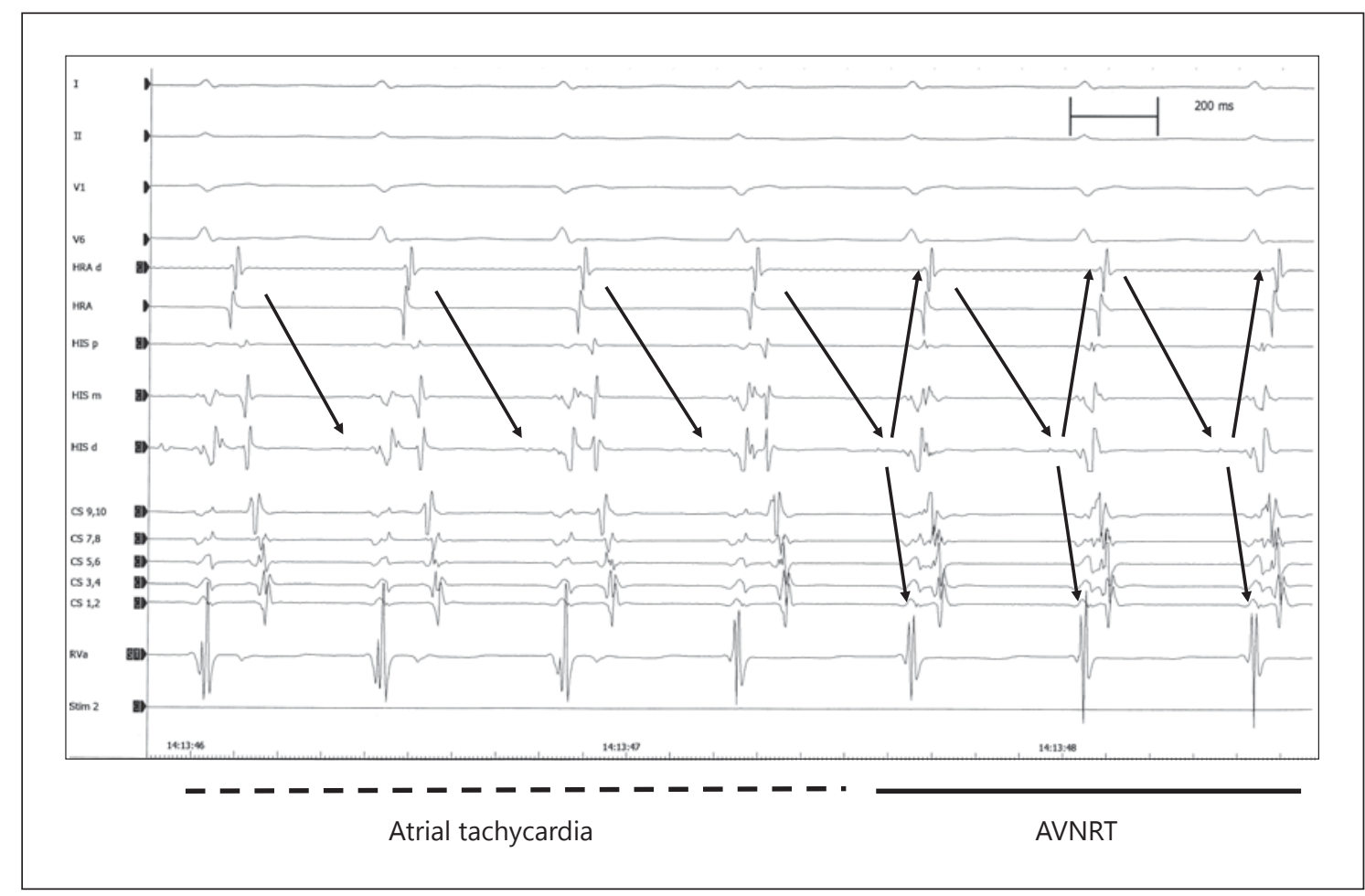

Fig. 2. Spontaneous transition from atrial tachycardia (CL $420 \mathrm{~ms}$ ) to typical AVNRT (CL $370 \mathrm{~ms}$ ). Surface leads and intracardiac electrogram recordings are arranged identically to figure 1 . Please note that the right atrial tachycardia is conducted in a 1:1 fashion to the ventricle over the slow pathway serving as a prerequisite for transition to AVNRT (for abbreviations, see legend to fig. 1).
Table 3. Transitions from AVNRT to another type of arrhythmia and vice versa during electrophysiological testing $(n=25 ; 5 \%)$

Transition of arrhythmias

$\mathrm{n}$

AVNRT to

Atrial fibrillation

10

Atrial tachycardia

8

Atrioventricular reciprocating tachycardia

Atrial flutter

Right ventricular outflow tract tachycardia/extrasystole

tachycardias $(\mathrm{n}=57,12 \%)$. Three different types of arrhythmias were encountered in 46 (9\%) patients, 4 kinds were found in $10(2 \%)$ patients, and 1 patient presented even 5 different forms of supraventricular arrhythmias during EPS. Transitions between ongoing AVNRT and other forms of tachycardia occurred in 25 (5\%) patients (table 3). A direct transition to or from AF was observed in 10 patients (fig. 1), atrial tachycardia was seen in 8 patients (fig. 2), AV reciprocating tachycardia was found in

4 patients, atrial flutter was observed in 2 cases, and right ventricular outflow tract tachycardia was seen in 1 case (fig. 3). Catecholamine was used less frequently in patients with more than one inducible arrhythmia (56 of $198 ; 28.3 \%)$ than in patients with AVNRT only (117 of $295 ; 40 \%)$.

All patients with AVNRT were treated successfully with radiofrequency ablation according to standard criteria of noninducibility with pharmacological provocation. Postablational EP testing revealed modification of the slow pathway in $295(60 \%)$ patients and complete elimination of the slow pathway in 198 (40\%) patients. Two or more coexisting tachycardias were ablated in the same procedure in 29 (6\%) patients. The most common targets were AVNRT and AV reciprocating tachycardia related to a concealed accessory pathway. Ablation of atrial tachycardia or flutter in the same procedure was performed less frequently and only if considered clinically relevant. Idiopathic right ventricular outflow tract tachycardia was ablated together with AVNRT in only 1 case. A separate EPS was scheduled in 13 (3\%) patients in order to ablate complex atrial arrhythmias or outflow 


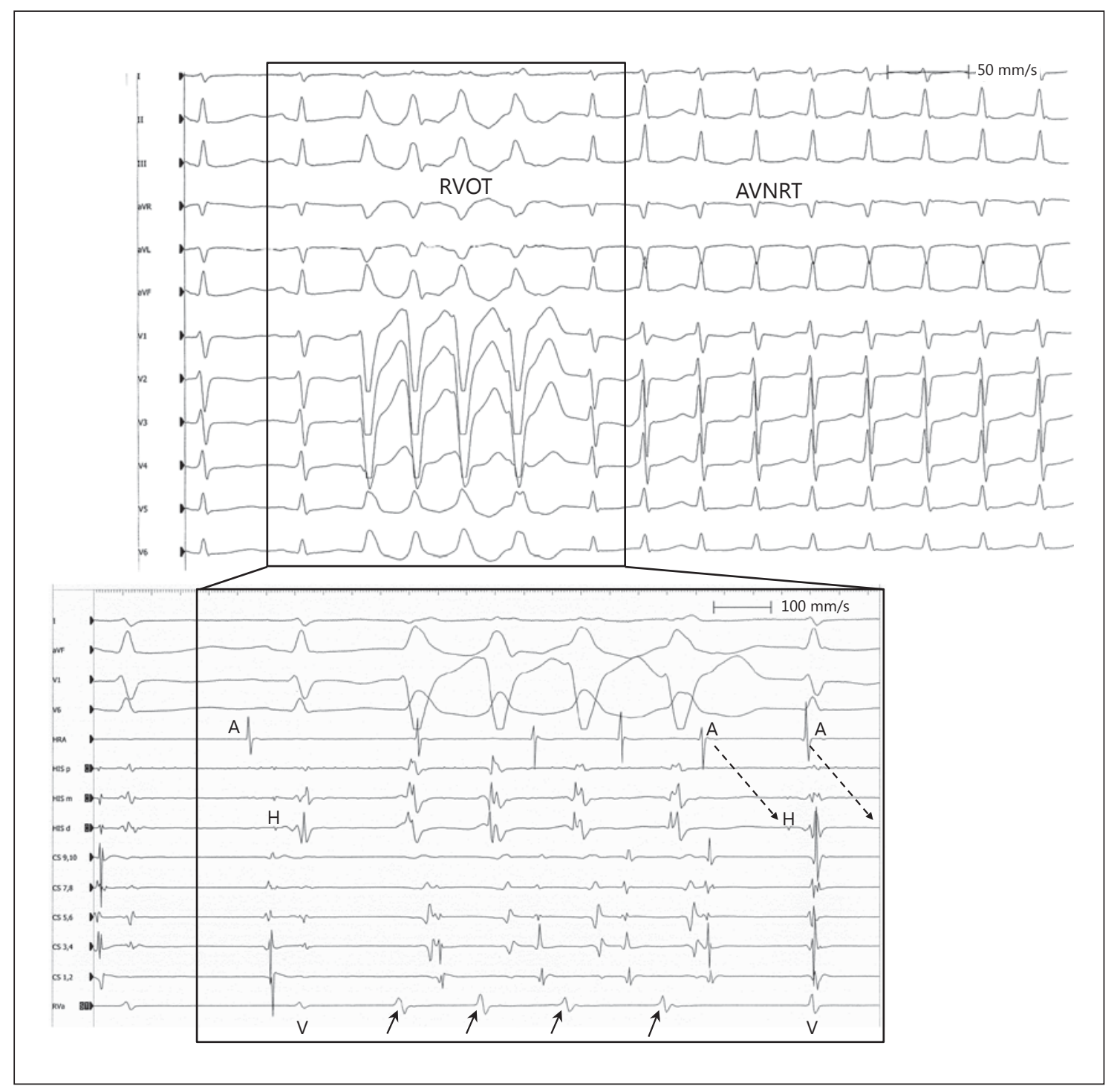

Fig. 3. Spontaneous onset of nonsustained idiopathic ventricular tachycardia originating from the right ventricular outflow tract (RVOT) with transition to or initiation of typical AVNRT. The two different arrhythmias are displayed on a 12-lead ECG on top with the intracardiac recordings during electrophysiological testing be- low. A normal sinus beat is followed by four repetitive ectopic ventricular depolarizations from the RVOT (small arrows) with varying VA intervals, finally initiating typical AVNRT via conduction over the slow pathway (dashed arrows) (for abbreviations, see legend to fig. 1). tract tachycardia in a dedicated procedure. Overall, a 3 -dimensional mapping system was used in 22 cases to reveal the exact mechanism and to guide ablation.

After hospital discharge, all patients were scheduled for the first follow-up visit in our outpatient clinic. A total of 443 (90\%) patients showed up for this appointment at our institution. The clinical outcome characteristics over the course of a short-term follow-up are summarized in table 4 . The median time to the first follow-up was 80 days. Two thirds of the patients were free of symptoms $(\mathrm{n}=313 ; 64 \%)$; however, $130(26 \%)$ reported a variety of perceptions, such as palpitations $(n=85)$, nonsustained arrhythmia $(\mathrm{n}=22)$, sustained arrhythmia $(\mathrm{n}=15)$, vertigo $(\mathrm{n}=4)$, angina-like thorax sensations $(\mathrm{n}=2)$, syncope $(\mathrm{n}=1)$, and dyspnea $(\mathrm{n}=1)$. Among the group of symptomatic individuals, 71 (55\%) patients were diagnosed with AVNRT only, whereas the remaining 59 (45\%) were diagnosed with more than one inducible ar- 
Table 4. Clinical outcome at the first follow-up: comparison of patients $(n=130)$ with AVNRT only versus AVNRT with one or more inducible arrhythmias at the initial EPS

\begin{tabular}{lrc}
\hline Clinical events and outcome & $\begin{array}{c}\text { AVNRT only } \\
(\mathrm{n}=71 ; 55 \%)\end{array}$ & $\begin{array}{l}\text { AVNRT with } \\
\text { one or more } \\
\text { inducible } \\
\text { arrhythmias } \\
(\mathrm{n}=59 ; 45 \%)\end{array}$ \\
& & 33 \\
\hline Palpitations & 52 & 2 \\
'Start-like' & 7 & 28 \\
Ectopics & 34 & 3 \\
Fast heart rate & 11 & 12 \\
Nonsustained arrhythmias & 10 & 12 \\
Sustained arrhythmias & 3 & 24 \\
ECG/external event recording & 18 & 3 \\
$\quad$ Sinus tachycardia & 16 & 8 \\
$\quad$ Atrial tachycardia & 0 & 8 \\
Atrial flutter & 0 & 2 \\
Atrial fibrillation & 0 & 10 \\
Ventricular ectopics & 2 & 0 \\
Vertigo & 4 & 1 \\
Syncope & 0 & 1 \\
Dyspnea & 0 & 23 \\
Antiarrhythmic drugs & 12 & 2 \\
Redo EPS & & 5 \\
For recurrent AVNRT & 0 & 2 \\
For other arrhythmias & 0 & \\
Other treatment & 2 & \\
\hline
\end{tabular}

rhythmia during EPS ( $\mathrm{p}=$ n.s.). ECG documentation due to palpitations was achieved in 18 patients with AVNRT only and in 24 patients with one or more inducible arrhythmias mostly in accordance with the additionally induced arrhythmia. About a quarter $(\mathrm{n}=35,27 \%)$ of the symptomatic patients were treated with medication, and only 5 patients with one or more inducible arrhythmias were willing to undergo a redo procedure.

Most of the patients who were symptomatic at the first follow-up ( $\mathrm{n}=102 ; 78 \%$ ) were invited for a second control visit at our outpatient clinic. The median follow-up time was 16 months. One third $(\mathrm{n}=35 ; 34 \%)$ of these patients had a diagnosis of AVNRT only, and two thirds $(\mathrm{n}=67 ; 66 \%)$ had more than 2 different types of arrhythmias during the initial EPS ( $\mathrm{p}=$ n.s.). A symptom-rhythm correlation was achieved in 12 patients with AVNRT only and in 19 patients with one or more inducible arrhythmias. Only few patients were symptomatic due to paroxysmal $(n=6)$ or persistent $(n=3)$ AF. Two thirds $(n=35)$ of the symptomatic patients during long-term follow-up were treated with antiarrhythmic drugs, 4 patients under- went cardioversion of AF, 12 were scheduled for a redo procedure, and 1 was implanted with a pacemaker due to documented asystole.

\section{Discussion}

Coexistence of AVNRT with other types of arrhythmias may be an underappreciated phenomenon in clinical practice for various reasons. The present study showed that coexistence of AVNRT with other SVT was a common finding among the typical referral population and posed some procedural as well as clinical challenges for the responsible electrophysiologist.

The most frequently observed double tachycardia was the combination of AVNRT with AF. The coexistence of AVNRT with AF has been reported previously, and AVNRT may even play a trigger role in AF $[1,2,14]$. Sauer et al. [2] identified AVNRT as a trigger for AF in a small young patient cohort and found an improved success rate for the cure of AF with additional slow-pathway modification. They hypothesized that AVNRT ablation may be the only procedure necessary for the cure of AF in selected patients. As the coexistence of AVNRT and AF seems to be frequent, screening for AVNRT or a concealed accessory pathway is considered helpful in younger patients undergoing ablation of AF. In a few cases in the study population, AF was triggered by AVNRT or other types of supraventricular arrhythmias warranting ablation of AVNRT and/ or non-PV triggers as an initial attempt. Moreover, patients and referring doctors should be alert to irregular arrhythmias after successful AVNRT ablation as these may have a significant influence on further management regarding the need for oral anticoagulation, antiarrhythmic drug management, or a left atrial ablation procedure. Despite the relatively high incidence of pace-induced AF in the present study, only a few patients developed clinically relevant atrial tachyarrhythmias, such as AF or flutter, during follow-up. Atrial tachyarrhythmias were considered to be clinically important if repetitive episodes lasted longer than $30 \mathrm{~s}$ as documented by ECG and were clearly associated with symptoms or hemodynamic compromise. In the setting of EP testing, AF is frequently a nonspecific phenomenon and is sometimes related to aggressive pacing techniques on catecholamines. Without a doubt, it is helpful for the clinician to obtain detailed information about the various types of arrhythmias induced at the time of EPS, as any coexisting arrhythmia may become clinically relevant after elimination of AVNRT. 
In a prospective study, Sticherling et al. [3] induced atrial tachycardia in $15 \%$ of patients who underwent slow-pathway ablation for AVNRT. Only 7\% of patients developed symptoms attributable to atrial tachycardia later on. In the present study, the incidence of a concomitant focal atrial tachycardia was clearly less frequent ( $\mathrm{n}=$ 40; 8\%). However, during follow-up, symptomatic atrial tachycardia was documented in 13 patients with a prior electrophysiological diagnosis of atrial tachycardia and this turned out to be helpful for the clinical decision to ablate this arrhythmia in a separate procedure. Our data are in line with previous studies which demonstrated a low incidence of recurrent palpitations attributable to atrial tachycardia after catheter ablation of AVNRT.

The coexistence of AVNRT with an accessory pathway has been described earlier and provides the substrate for various combinations and interactions $[4,15]$. In the present study, the most common targets for ablation were the slow pathway and a concealed paranodal bypass tract serving as substrate for micro- and macroreentry tachycardias. Ablation of both types of arrhythmias is usually feasible and safe in the same procedure, particularly if there is some spatial distance. However, simultaneous ablation of double tachycardias in one session remains a controversial issue for multiple reasons. In the rare instance of an anteroseptal accessory pathway, it may be prudent to avoid slow-pathway elimination ahead of targeting of a bypass tract in proximity to the fast pathway. Similarly, septal AT arising from the perinodal tissue or the aortic root is sometimes difficult to differentiate from AVNRT as separate arrhythmia and ablation might be harmful if multiple AV nodal inputs are ablated in one procedure.

Kuo et al. [16] described a transition between AVNRT and AVRT in 3 individuals and between AVNRT and atrial tachycardia in 5 individuals observed in a small cohort of 35 patients with double inducible tachycardias. In contrast to that, our data documented a frequency of $5 \%$ for transitions between AVNRT and other types of tachycardias. The most frequent transition was observed between AVNRT and AF, followed by atrial tachycardia and AV reciprocating tachycardia. Due to the incidental phenomenon of transition between AVNRT and other types of SVT, it may be speculated upon the mechanism of tachycardia-induced tachycardia. It is of interest that some coexisting arrhythmias were no longer inducible after successful slow-pathway ablation.

A coincidence of idiopathic outflow tract ventricular tachycardia and AVNRT was found in 15\% of cases of clinically documented idiopathic ventricular tachycardia by Kautzner et al. [5]. A clear association between these two differently located arrhythmia substrates has been reported also by other groups $[6,7]$. Among our study population, the simultaneous occurrence of AVNRT with ventricular outflow tract extrasystole and/or tachycardia was observed with a rather low incidence (4\%). Consequently, idiopathic ventricular tachycardia was ablated in addition to AVNRT during the same procedure in only a few highly symptomatic patients. The majority of patients with ventricular outflow tract ectopics were treated medically or received reassurance about it being harmless in the absence of structural heart disease.

The majority of patients were free of symptoms after ablation of AVNRT at the short-term follow-up; however, one third of the patients reported a variety of symptoms. With respect to palpitations, the outcomes were not different between individuals with AVNRT only and patients with AVNRT plus a coexisting arrhythmia. Importantly, event recording documented a higher frequency of sinus tachycardia as a correlate of the reported palpitations among group 1, whereas a higher prevalence of atrial tachyarrhythmias was the reason in group 2 . The latter group more often required antiarrhythmic drug therapy as well as redo interventions later on.

Frequent documentation of AVNRT with other arrhythmias, particularly SVT, may imply that this is not just a random coexistence. However, most arrhythmias have different mechanisms and the relationship appears to be more or less incidental. The exact mechanism of AVNRT remains elusive; however, some interactions or interdependence may exist between atrial myocardium and AV nodal or perinodal tissue [17].

The major limitation of this study was its retrospective design. The definition of a coexisting arrhythmia lasting for at least $6 \mathrm{~s}$ was chosen arbitrarily rather than based on the usual definition of a sustained arrhythmia.

\section{Conclusion}

The present study demonstrated that a variety of arrhythmias were encountered in patients with AVNRT. The most frequently observed double tachycardia was the combination of AVNRT with AF. However, it has to be carefully determined whether concomitant atrial tachyarrhythmias are of clinical significance for further patient management or a nonspecific finding related to pacing maneuvers on pharmacological provocation. Clinical outcomes with respect to palpitations deserving medical therapy or additional interventions after ablation of AVNRT may be explained by other coexisting arrhythmias. 


\section{References}

1 Hamer ME, Wilkinson WE, Clair WK, et al: Incidence of symptomatic atrial fibrillation in patients with paroxysmal supraventricular tachycardia. J Am Coll Cardiol 1995;25:984988.

-2 Sauer WH, Alonso C, Zado E, et al: Atrioventricular nodal reentrant tachycardia in patients referred for atrial fibrillation ablation: response to ablation that incorporates slowpathway modification. Circulation 2006;114: 191-195.

$>3$ Sticherling C, Tada H, Greenstein R, et al: Incidence and clinical significance of inducible atrial tachycardia in patients with atrioventricular nodal reentrant tachycardia. J Cardiovasc Electrophysiol 2001;12:507-510.

-4 Zardini M, Leitch JW, Guiraudon GM, et al: Atrioventricular nodal reentry and dual atrioventricular node physiology in patients undergoing accessory pathway ablation. Am J Cardiol 1990;66:1388-1389.

5 Kautzner J, Cihák R, Vancura V, et al: Coincidence of idiopathic ventricular outflow tract tachycardia and atrioventricular nodal reentrant tachycardia. Europace 2003;5:215-220.

6 Wylie JV Jr, Milliez P, Germano JJ, et al: Atrioventricular nodal reentrant tachycardia associated with idiopathic ventricular tachycardia: clinical and electrophysiologic characteristics. J Electrocardiol 2007;40:94-99.
7 Ozin B, Pirat B, Muderrisoglu H: Interesting electrophysiological findings in a patient with coincidental right ventricular outflow tract and atrioventricular nodal reentrant tachycardia. Indian Pacing Electrophysiol J 2004;4: 93-96.

$\checkmark 8$ Chen SA, Chiang CE, Yang CJ, et al: Radiofrequency catheter ablation of sustained intraatrial reentrant tachycardia in adult patients: identification of electrophysiological characteristics and endocardial mapping techniques. Circulation 1993;88:578-587.

$\checkmark 9$ Katritsis DG, Camm AJ: Atrioventricular nodal reentrant tachycardia. Circulation 2010;122:831-840.

10 Katritsis DG, Josephson ME: Classification of electrophysiological types of atrioventricular nodal re-entrant tachycardia: a reappraisal. Europace 2013;15:1231-1240.

11 Jackman WM, Wang XZ, Friday KJ, et al: Catheter ablation of accessory atrioventricular pathways (Wolff-Parkinson-White syndrome) by radiofrequency current. N Engl J Med 1991;324:1605-1611.

12 Jackman WM, Beckman KJ, McClelland JH, et al: Treatment of supraventricular tachycardia due to atrioventricular nodal reentry, by radiofrequency catheter ablation of slowpathway conduction. N Engl J Med 1992;327: 313-318.
13 Haissaguerre M, Gaita F, Fischer B, et al: Elimination of atrioventricular nodal reentrant tachycardia using discrete slow potentials to guide application of radiofrequency energy. Circulation 1992;85:2162-2175.

14 Chen YJ, Tai CT, Hsieh MH, et al: Dependence of electrogram duration in right posteroseptal atrium and atrium-pulmonary vein junction on pacing site: mechanism and implications regarding atrioventricular nodal reentrant tachycardia and paroxysmal atrial fibrillation. J Cardiovasc Electrophysiol 2000; 11:506-515.

15 Delacretaz E, Soejima K, Stevenson WG, et al: Short ventriculoatrial intervals during orthodromic atrioventricular reciprocating tachycardia: what is the mechanism? J Cardiovasc Electrophysiol 2000;11:121-124.

-16 Kuo JY, Tai CT, Chiang CE, et al: Mechanisms of transition between double paroxysmal supraventricular tachycardias. J Cardiovasc Electrophysiol 2001;12:1339-1345.

17 Mendenhall GS, Voigt A, Saba S: Insights into atrioventricular nodal function from patients displaying dual conduction properties: interactive and orthogonalpathways. Circ Arrhythm Electrophysiol 2013;6:364-370. 